\title{
On the meromorphic solutions of certain class of nonlinear differential equations
}

\author{
Nana Liu', Weiran Lü ${ }^{1 *}$ and Chungchun Yang ${ }^{2}$ \\ Dedicated to Professor George Csordas on the occasion of his retirement.
}

"Correspondence: luwr@upc.edu.cn ${ }^{1}$ Department of Mathematics, China University of Petroleum, Qingdao, 266580, P.R. China

Full list of author information is available at the end of the article

\begin{abstract}
Let $\alpha$ be an entire function, $a_{n-1}, \ldots, a_{1}, a_{0}, R$ be small functions of $f$, and let $n \geq 2$ be an integer. Then, for any positive integer $k$, the differential equation $f^{n} f^{(k)}+a_{n-1} f^{n-1}+\cdots+a_{1} f+a_{0}=R \mathbf{e}^{\alpha}$ has transcendental meromorphic solutions under appropriate conditions on the coefficients. In addition, for $n=1$ and $k=1$, we have extended some well-known and relevant results obtained by others, by using different arguments.
\end{abstract}

MSC: 34M10; 30D35

Keywords: entire function; differential polynomial; Nevanlinna theory; Hayman's alternative; differential equation

\section{Introduction and main results}

In this paper, a meromorphic function means meromorphic in the whole complex plane. We shall adopt the standard notations in Nevanlinna's value distribution theory of meromorphic functions (see, e.g., [1, 2]).

Given a meromorphic function $f$, recall that $\alpha \not \equiv 0, \infty$ is a small function with respect to $f$, if $T(r, \alpha)=S(r, f)$, where $S(r, f)$ denotes any quantity satisfying $S(r, f)=o\{T(r, f)\}$ as $r \rightarrow \infty$, possibly outside a set of $r$ of finite linear measure.

Theorem A Let $f$ be a transcendental meromorphic function, $n(\geq 3)$ be an integer. Then $F=f^{n} f^{\prime}$ assumes all finite values, except possibly zero, infinitely many times.

The above theorem was derived by Hayman [3] in 1959. Later, he conjectured [4] that Theorem A remains valid even if $n=1$ or $n=2$. Mues [5] proved the result for $n=2$ and the case $n=1$ was proved by Bergweiler and Eremenko [6] and independently by Chen and Fang [7]. For entire functions and difference polynomials, similar results have been obtained by others earlier (see, e.g., [8-11]).

Theorem $\mathbf{B}$ ([12]) Iff is a transcendental meromorphic function of finite order and a $(\not \equiv 0)$ is a polynomial, then $f^{\prime}-$ a has infinitely many zeros.

Wang [13] obtained the following result.

(c) 2015 Liu et al.; licensee Springer. This article is distributed under the terms of the Creative Commons Attribution 4.0 International License (http://creativecommons.org/licenses/by/4.0/), which permits unrestricted use, distribution, and reproduction in any medium, provided you give appropriate credit to the original author(s) and the source, provide a link to the Creative Commons license, and indicate if changes were made. 
Theorem $\mathrm{C}$ Let $f$ be a transcendental entire function and $n, k$ be positive integers, and let $c(z)(\not \equiv 0)$ be a small function with respect to $f$. If $T(r, f) \neq \tau N_{1)}(r, 1 / f)+S(r, f)$, then $f^{n}(z) f^{(k)}(z)-c(z)$ has infinitely many zeros, where $\tau=0$ if $n \geq 2$ or $k=1 ; \tau=1$ otherwise.

In this paper, by using methods different from that were used by others (see, e.g., [10, 14] and [15]), we shall extend and generalize the above results with $f^{n} f^{(k)}$ being replaced by a differential polynomial $P_{n+1}(f)$. Specifically, our main results can be stated as follows.

Theorem 1.1 Let $\alpha$ be an entire function, $R$ and $a_{i}(i=0,1, \ldots, n-1)$ be small functions of $f$ with $a_{0} \not \equiv$. If, for $n \geq 2$, a transcendental meromorphic function $f$ satisfies the differential equation

$$
f^{n} f^{(k)}+a_{n-1} f^{n-1}+\cdots+a_{1} f+a_{0}=R \mathrm{e}^{\alpha},
$$

then, for any positive integer $k$, we have $f=g \exp (\alpha /(n+1))-(n+1) \frac{a_{0}}{a_{1}}$ with $g^{n+1}=$ $\left[\frac{(n+1) a_{0}}{a_{1}}\right]^{n+1} \frac{R}{a_{0}}$, and $\left(\frac{a_{0}}{a_{1}}\right)^{(k)}+\frac{n}{n+1}\left(\frac{1}{n+1} \frac{a_{1}}{a_{0}}\right)^{n} a_{0} \equiv 0$.

Remark 1.1 Let $a_{0}$ and $a_{1}$ be non-zero constants in Theorem 1.1. Then (1.1) has no transcendental meromorphic solutions.

A meromorphic solution $f$ of (1.1) is called admissible, if $T\left(r, \alpha_{j}\right)=S(r, f)$ holds for all coefficients $\alpha_{j}(j=0, \ldots, n-1)$ and $T(r, R)=S(r, f)$.

Remark 1.2 If $a_{0} \equiv 0$ and $n \geq 2, k \geq 1$, then the other coefficients $a_{1}, \ldots, a_{n-1}$ must be identically zero. In this case, (1.1) becomes $f^{n} f^{(k)}=R \mathrm{e}^{\alpha}$ and $f$ has the form $f=u \exp (\alpha /(n+$ $1)$ ) as the only possible admissible solution of (1.1), where $u$ is a small function of $f$.

We have the following corollary by Theorem 1.1.

Corollary 1.1 Let $f$ be a transcendental meromorphic function with $N(r, f)=S(r, f), n \geq 2$ be an integer. If $\left(\frac{a_{0}}{a_{1}}\right)^{(k)}+\frac{n}{n+1}\left(\frac{1}{n+1} \frac{a_{1}}{a_{0}}\right)^{n} a_{0} \not \equiv 0$, then $F=f^{n} f^{(k)}+a_{n-1} f^{n-1}+\cdots+a_{1} f+a_{0}$ has infinitely many zeros, where $a_{i}(i=0,1, \ldots, n-1)$ are small functions off such that $a_{0} \not \equiv 0$.

Note that in Theorem 1.1, it is assumed that $n \geq 2$ and $k \geq 1$. However, for $n=1$ and $k=1$, we can derive the following result.

Theorem 1.2 Let $p, q$, and $R$ be non-zero polynomials, $\alpha$ be an entire function. Then the differential equation $p f^{\prime}-q=\mathrm{e}^{\alpha}$ has no transcendental meromorphic solutions, where $p$, $q$, and $R$ are small functions off with $p q \neq \equiv$.

Remark 1.3 From the proof of Theorem 1.2, we see that the restriction in Theorem 1.2 to $p, q$, and $R$ may extend to small functions. In fact, it is easy to find that the conclusion is valid provided that $p, q$, and $R$ are non-vanishing small functions of $f$. The following corollary arises directly from an immediate consequence of Theorem 1.2.

Corollary 1.2 Let $f$ be a transcendental meromorphic function with $N(r, f)=S(r, f), p$ and $q$ be non-vanishing small functions off. Then $F=p f f^{\prime}-q$ has infinitely many zeros. 


\section{Some lemmas and proofs of theorems}

In order to prove our conclusions, we need some lemmas. The following lemma is fundamental to Clunie's theorem [16].

Lemma 2.1 $([17,18])$ Let $f$ be a transcendental meromorphic solution of

$$
f^{n} P(z, f)=Q(z, f)
$$

where $P(z, f)$ and $Q(z, f)$ are polynomials in $f$ and its derivatives with meromorphic coefficients $\left\{a_{\lambda} \mid \lambda \in I\right\}$ such that $m\left(r, a_{\lambda}\right)=S(r, f)$ for all $r \in I$. If the total degree of $Q(z, f)$ as a polynomial in $f$ and its derivatives is less than or equal to $n$, then $m(r, P(r, f))=S(r, f)$.

The following lemma is crucial to the proof of our theorems.

Lemma 2.2 $([18,19])$ Let $f$ be a meromorphic solution of an algebraic equation

$$
P\left(z, f, f^{\prime}, \ldots, f^{(n)}\right)=0
$$

where $P$ is a polynomial in $f, f^{\prime}, \ldots, f^{(n)}$ with meromorphic coefficients small with respect to $f$. If a complex constant $c$ does not satisfy (2.1), then

$$
m\left(r, \frac{1}{f-c}\right)=S(r, f)
$$

Proof of Theorem 1.1 Let $f$ be a transcendental meromorphic function that satisfies (1.1). Then two cases are to be treated, namely case $1: N(r, f) \neq S(r, f)$, and case $2: N(r, f)=S(r, f)$. For case 1 , it is impossible as $\alpha$ is an entire function and $R, a_{1}, \ldots, a_{n}$ are small functions of $f$.

To prove Theorem 1.1, we now suppose that $N(r, f)=S(r, f)$.

Denoting $\phi:=f^{n} f^{(k)}+a_{n-1} f^{n-1}+\cdots+a_{1} f$, and assuming that $T(r, \phi)=S(r, f)$, then by Lemma 2.1, we get $m\left(r, f^{(k)}\right)=S(r, f)$ and then $T\left(r, f^{(k)}\right)=S(r, f)$, since $N(r, f)=S(r, f)$ by the assumption. The contradiction $T(r, f)=S(r, f)$ now follows by the theorem in [20] and combining it with the proof of Proposition E in [21]. Thus, for any transcendental meromorphic function $f$ under the condition: $N(r, f)=S(r, f)$,

$$
T\left(r, f^{n} f^{(k)}+a_{n-1} f^{n-1}+\cdots+a_{1} f\right) \neq S(r, f) .
$$

From (1.1) and the result of Milloux (see, e.g., [1], Theorem 3.1), one can easily show that

$$
T\left(r, \mathrm{e}^{\alpha}\right) \leq(n+1) T(r, f)+S(r, f),
$$

which leads to $T(r, \alpha)+T\left(r, \alpha^{\prime}\right)=S(r, f)$.

By taking the logarithmic derivative on both sides of (1.1), we have

$$
\frac{n f^{n-1} f^{\prime} f^{(k)}+f^{n} f^{(k+1)}+a_{n-1}^{\prime} f^{n-1}+\cdots+a_{1}^{\prime} f+a_{1} f^{\prime}+a_{0}^{\prime}}{f^{n} f^{(k)}+a_{n-1} f^{n-1}+\cdots+a_{1} f+a_{0}}=\frac{R^{\prime}}{R}+\alpha^{\prime} .
$$


It follows by (2.3) that

$$
\begin{aligned}
& -\left(\frac{R^{\prime}}{R}+\alpha^{\prime}\right) f^{n} f^{(k)}+n f^{n-1} f^{\prime} f^{(k)}+f^{n} f^{(k+1)}+\left\{a_{n-1}^{\prime}-\left(\frac{R^{\prime}}{R}+\alpha^{\prime}\right) a_{n-1}\right\} f^{n-1} \\
& +(n-1) a_{n-1} f^{n-2} f^{\prime}+\cdots+\left\{a_{1}^{\prime}-\left(\frac{R^{\prime}}{R}+\alpha^{\prime}\right) a_{1}\right\} f+a_{1} f^{\prime}=\left(\frac{R^{\prime}}{R}+\alpha^{\prime}\right) a_{0}-a_{0}^{\prime} .
\end{aligned}
$$

If $\left(\frac{R^{\prime}}{R}+\alpha^{\prime}\right) a_{0}-a_{0}^{\prime} \equiv 0$, then $A a_{0}=R e^{\alpha}$, where $A$ is a non-zero constant. From (1.1), we get

$$
f^{n} f^{(k)}+a_{n-1} f^{n-1}+\cdots+a_{1} f=(A-1) a_{0} .
$$

If $A=1$, then from (2.5), we obtain

$$
f^{n} f^{(k)}+a_{n-1} f^{n-1}+\cdots+a_{1} f \equiv 0
$$

which contradicts (2.2). However, if $A \neq 1$, then again from (2.5), we would derive

$$
T\left(r, f^{n} f^{(k)}+a_{n-1} f^{n-1}+\cdots+a_{1} f\right)=S(r, f)
$$

a contradiction.

Thus

$$
\left(\frac{R^{\prime}}{R}+\alpha^{\prime}\right) a_{0}-a_{0}^{\prime}:=\varphi \not \equiv 0
$$

In this case, from (2.4), we have

$$
N_{(2}\left(r, \frac{1}{f}\right) \leq N\left(r, \frac{1}{\varphi}\right)+S(r, f) \leq T(r, \varphi)+S(r, f)=S(r, f),
$$

where $N_{(2}\left(r, \frac{1}{f}\right)$, as usually, denotes the counting function of zeros of $f$ whose multiplicities are not less than 2 , which implies that the zeros of $f$ are mainly simple zeros. Again, from (2.4), the fact that $\alpha^{\prime}$ is a small function of $f$ and Lemma 2.2 (where $c=0$ is used), we conclude $m\left(r, \frac{1}{f}\right)=S(r, f)$. This together with Nevanlinna's first theorem will result in

$$
T(r, f)=N\left(r, \frac{1}{f}\right)+S(r, f)=N_{1)}\left(r, \frac{1}{f}\right)+S(r, f),
$$

where in $N_{1)}(r, 1 / f)$ only the simple zeros of $f$ are to be considered.

Assume that $a_{1} \equiv 0$. It follows by (2.4) and $n \geq 2$ that $N_{1)}(r, 1 / f)=S(r, f)$, which contradicts (2.6). Thus $a_{1} \not \equiv 0$. Let $z_{0}$ be a simple zero of $f$, and $z_{0}$ be not a pole of one of the coefficients $a_{i},\left(\frac{R^{\prime}}{R}+\alpha^{\prime}\right) a_{i}-a_{i}^{\prime}(i=1,2, \ldots, n-1)$. From (2.4), we see that $z_{0}$ is a zero of $a_{1} f^{\prime}+a_{0}^{\prime}-\left(\frac{R^{\prime}}{R}+\alpha^{\prime}\right) a_{0}$. Set

$$
h=\frac{a_{1} f^{\prime}+a_{0}^{\prime}-\left(\frac{R^{\prime}}{R}+\alpha^{\prime}\right) a_{0}}{f} .
$$

Then (2.7) gives $T(r, h)=S(r, f)$. We have

$$
f^{\prime}=\frac{1}{a_{1}}\left\{h f-a_{0}^{\prime}+\left(\frac{R^{\prime}}{R}+\alpha^{\prime}\right) a_{0}\right\}:=\mu_{1} f+\nu_{1} .
$$


Clearly, it follows from $(2.6)$ and $T\left(r, \mu_{1}\right)+T\left(r, v_{1}\right)=S(r, f)$ that $\mu_{1} v_{1} \not \equiv 0$. By (2.3), we obtain

$$
f^{n-1} \psi=P_{n-1}(f)
$$

where $\psi=-\left(\frac{R^{\prime}}{R}+\alpha^{\prime}\right) f^{(k)}+n f^{\prime} f^{(k)}+f^{(k+1)}, P_{n-1}(f)=\left(\frac{R^{\prime}}{R}+\alpha^{\prime}\right)\left(a_{n-1} f^{n-1}+\cdots+a_{1} f+a_{0}\right)-$ $\left(a_{n-1} f^{n-1}+\cdots+a_{1} f+a_{0}\right)^{\prime}$. It follows by $(2.2)$ that $P_{n-1}(f) \not \equiv 0$. Thus $\psi \not \equiv 0$. Moreover, by applying Lemma 2.1 to (2.9), we get $m(r, \psi)=S(r, f)$. It is easy to see by $N(r, f)=S(r, f)$ that $T(r, \psi)=S(r, f)$.

From (2.8) and induction, we have $f^{\prime \prime}=\left(\mu_{1}^{\prime}+\mu_{1}^{2}\right) f+\mu_{1} v_{1}+v_{1}^{\prime}:=\mu_{2} f+v_{2}$, and

$$
f^{(k)}=\mu_{k} f+v_{k}
$$

where $\mu_{k}, v_{k}$ are small functions of $f$. By the expression of $\psi$ and (2.6), we get $v_{k} \not \equiv 0$. If $\mu_{k} \equiv 0$, then $(2.10)$ gives $T\left(r, f^{(k)}\right)=S(r, f)$, which is impossible. Therefore, $\mu_{k} \not \equiv 0$.

By (2.10), (1.1) becomes

$$
\mu_{k} f^{n+1}+v_{k} f^{n}+a_{n-1} f^{n-1}+\cdots+a_{1} f+a_{0}=\mathrm{Re}^{\alpha} .
$$

By applying the Tumura-Clunie lemma (see, e.g., [1], Theorem 3.9) to the left-hand side of (2.11), we have $\mu_{k}\left[f+\frac{v_{k}}{(n+1) \mu_{k}}\right]^{n+1}=R \mathrm{e}^{\alpha}$ and $f=g \mathrm{e}^{\alpha /(n+1)}-\frac{v_{k}}{(n+1) \mu_{k}}$ with $g^{n+1}=\frac{R}{\mu_{k}}$.

In view of (2.11), we have

$$
\mu_{k} f^{n+1}+v_{k} f^{n}+a_{n-1} f^{n-1}+\cdots+a_{1} f+a_{0}=\mu_{k}\left[f+\frac{v_{k}}{(n+1) \mu_{k}}\right]^{n+1} .
$$

Thus, we have

$$
\frac{1}{n+1} \frac{\nu_{k}}{\mu_{k}}=(n+1) \frac{a_{0}}{a_{1}} \quad \text { and } \quad \mu_{k}=\left(\frac{1}{n+1} \frac{a_{1}}{a_{0}}\right)^{n+1} a_{0}
$$

By (2.12), we obtain $v_{k}=(n+1)\left(\frac{1}{n+1} \frac{a_{1}}{a_{0}}\right)^{n} a_{0}$ and $g^{n+1}=\left[\frac{(n+1) a_{0}}{a_{1}}\right]^{n+1} \frac{R}{a_{0}}$.

Set $(n+1) \gamma=\alpha$. It follows by $(2.10)$ and $f=g \mathrm{e}^{\gamma}-(n+1) \frac{a_{0}}{a_{1}}$ that

$$
f^{(k)}=\left(\frac{1}{n+1} \frac{a_{1}}{a_{0}}\right)^{n+1} a_{0}\left[g \mathrm{e}^{\gamma}-(n+1) \frac{a_{0}}{a_{1}}\right]+(n+1)\left(\frac{1}{n+1} \frac{a_{1}}{a_{0}}\right)^{n} a_{0} .
$$

In addition, by $f=g \mathrm{e}^{\gamma}-(n+1) \frac{a_{0}}{a_{1}}$ we get

$$
f^{(k)}=Q\left(g, g^{\prime}, \ldots, g^{(k)}\right) \mathrm{e}^{\gamma}-(n+1)\left(\frac{a_{0}}{a_{1}}\right)^{(k)}
$$

where $Q\left(g, g^{\prime}, \ldots, g^{(k)}\right)$ is a differential polynomial of $g$.

Thus, (2.13) and (2.14) imply

$$
Q\left(g, g^{\prime}, \ldots, g^{(k)}\right)=\left(\frac{1}{n+1} \frac{a_{1}}{a_{0}}\right)^{n+1} a_{0} g
$$


and

$$
(n+1)\left(\frac{a_{0}}{a_{1}}\right)^{(k)}=\left(\frac{1}{n+1} \frac{a_{1}}{a_{0}}\right)^{n+1} a_{0}(n+1) \frac{a_{0}}{a_{1}}-(n+1)\left(\frac{1}{n+1} \frac{a_{1}}{a_{0}}\right)^{n} a_{0} .
$$

It follows by $(2.15)$ that

$$
\left(\frac{a_{0}}{a_{1}}\right)^{(k)}+\frac{n}{n+1}\left(\frac{1}{n+1} \frac{a_{1}}{a_{0}}\right)^{n} a_{0}=0 .
$$

This completes the proof of Theorem 1.1.

Proof of Remark 1.2 Let $f$ be a transcendental meromorphic solution of (1.1). Since $a_{0} \equiv 0$, we have $N(r, 1 / f) \leq N(r, 1 / R)+S(r, f)=S(r, f)$. Obviously, $N(r, f)=S(r, f)$. In this case, there exist a meromorphic function $u$ and an entire function $v$ such that $f=u \mathrm{e}^{v}$, and $N(r, 1 / u)+N(r, u)=S(r, f)$. Clearly, from the expressions of $f$ and the Borel lemma (see, e.g., [2], Theorem 1.52), all the $a_{j}(j=1,2, \ldots, n-1)$ must be identically zero. Thus, Remark 1.2 follows.

Proof of Theorem 1.2 Now we proceed to prove the theorem by contradiction. Let $f$ be a transcendental meromorphic function that satisfies $p f f^{\prime}-q=\operatorname{Re}^{\alpha}$. Then two cases are to be retreated, namely $N(r, f) \neq S(r, f)$ and $N(r, f)=S(r, f)$. For $N(r, f) \neq S(r, f)$, this is impossible as $\alpha$ is an entire function and $R, p, q$ are non-zero polynomials.

To prove Theorem 1.2, we now suppose that $N(r, f)=S(r, f)$. We differentiate $p f^{\prime}-q=$ $\operatorname{Re}^{\alpha}$ and eliminate $\mathrm{e}^{\alpha}$,

$$
t_{1} f^{\prime}+p\left(f^{\prime}\right)^{2}+p f f^{\prime \prime}=t_{2}
$$

where $t_{1}=p^{\prime}-\left(\frac{R^{\prime}}{R}+\alpha^{\prime}\right) p, t_{2}=q^{\prime}-\left(\frac{R^{\prime}}{R}+\alpha^{\prime}\right) q$.

If $t_{2} \equiv 0$, then, by integrating the definition of $t_{2}, \alpha$ must be a constant, hence $f^{\prime}$ is rational, and then, by Lemma 2.1, $m\left(r, f^{\prime}\right)=S(r, f)$. Hence $T\left(r, f^{\prime}\right)=S(r, f)$. This is a contradiction by Proposition E in [21]. Thus, $t_{2} \not \equiv 0$, and then by (2.16), we get (2.6). By differentiating both sides of (2.16), we have

$$
t_{1}^{\prime} f^{\prime}+\left(t_{1}+p^{\prime}\right)\left(f^{\prime}\right)^{2}+\left(t_{1}+p^{\prime}\right) f^{\prime \prime}+3 p f^{\prime} f^{\prime \prime}+p f f^{\prime \prime \prime}=t_{2}^{\prime} .
$$

Letting $z_{0}$ be a simple zero of $f,(2.16)$ and (2.17) imply

$$
\left(p\left(f^{\prime}\right)^{2}-t_{2}\right)\left(z_{0}\right)=0
$$

and

$$
\left\{\left(t_{1}+p^{\prime}\right)\left(f^{\prime}\right)^{2}+3 p f^{\prime} f^{\prime \prime}-t_{2}^{\prime}\right\}\left(z_{0}\right)=0 \text {. }
$$

Let

$$
g=\frac{3 p t_{2} f^{\prime \prime}+\left[t_{2}\left(t_{1}+p^{\prime}\right)-t_{2}^{\prime} p\right] f^{\prime}}{f}
$$


From (2.6), (2.18), and (2.19), we get

$$
T(r, g)=S(r, f)
$$

By (2.20), we obtain

$$
f^{\prime \prime}=\alpha_{1} f+\beta_{1} f^{\prime}
$$

where

$$
\alpha_{1}=\frac{g}{3 p t_{2}}, \quad \beta_{1}=\frac{t_{2}^{\prime} p-t_{2}\left(t_{1}+p^{\prime}\right)}{3 p t_{2}}
$$

and

$$
T\left(r, \alpha_{1}\right)=S(r, f), \quad T\left(r, \beta_{1}\right)=S(r, f) .
$$

Substituting (2.21) into (2.16) yields

$$
\left(t_{1}+p \beta_{1}\right) f^{\prime}+p\left(f^{\prime}\right)^{2}+\alpha_{1} p f^{2}=t_{2} .
$$

On the other hand, from (2.21), we have

$$
f^{\prime \prime \prime}=\alpha_{2} f+\beta_{2} f^{\prime}
$$

where $\alpha_{2}=\alpha_{1}^{\prime}+\alpha_{1} \beta_{1}, \beta_{2}=\alpha_{1}+\beta_{1}^{\prime}+\beta_{1}^{2}$, and

$$
T\left(r, \alpha_{2}\right)=S(r, f), \quad T\left(r, \beta_{2}\right)=S(r, f) .
$$

Substituting (2.23) into (2.17), we have

$$
\left[t_{1}^{\prime}+\beta_{1}\left(t_{1}+p^{\prime}\right)+3 p \alpha_{1}+p \beta_{2}\right] f f^{\prime}+\left(t_{1}+p^{\prime}+3 p \beta_{1}\right)\left(f^{\prime}\right)^{2}+\left[\alpha_{1}\left(t_{1}+p^{\prime}\right)+\alpha_{2} p\right] f^{2}=t_{2}^{\prime} .
$$

It follows by (2.22) and (2.24) that

$$
\begin{aligned}
& \left\{p\left[t_{1}^{\prime}+\beta_{1}\left(t_{1}+p^{\prime}\right)+3 p \alpha_{1}+p \beta_{2}\right]-\left(t_{1}+p^{\prime}+3 p \beta_{1}\right)\left(t_{1}+p \beta_{1}\right)\right\} f^{\prime} \\
& \quad+\left\{p\left[\alpha_{1}\left(t_{1}+p^{\prime}\right)+\alpha_{2} p\right]-\alpha_{1} p\left(t_{1}+p^{\prime}+3 p \beta_{1}\right)\right\} f^{2}=t_{2}^{\prime} p-t_{2}\left(t_{1}+p^{\prime}+3 p \beta_{1}\right) .
\end{aligned}
$$

From the definition of $\beta_{1}$, we now claim $t_{2}^{\prime} p-t_{2}\left(t_{1}+p^{\prime}+3 p \beta_{1}\right) \equiv 0$. To show this, we assume the contrary, that is, $t_{2}^{\prime} p-t_{2}\left(t_{1}+p^{\prime}+3 p \beta_{1}\right) \not \equiv 0$. Then from the fact that $t_{2}^{\prime} p-$ $t_{2}\left(t_{1}+p^{\prime}+3 p \beta_{1}\right)$ is a small function of $f$ and (2.25), we get

$$
\begin{aligned}
N_{1)}\left(r, \frac{1}{f}\right) & \leq N\left(r, \frac{1}{t_{2}^{\prime} p-t_{2}\left(t_{1}+p^{\prime}+3 p \beta_{1}\right)}\right) \\
& \leq T\left(r, t_{2}^{\prime} p-t_{2}\left(t_{1}+p^{\prime}+3 p \beta_{1}\right)\right)+S(r, f)=S(r, f),
\end{aligned}
$$


and from this and (2.6) we deduce $T(r, f)=S(r, f)$, a contradiction. Thus, we have

$$
t_{2}^{\prime} p-t_{2}\left(t_{1}+p^{\prime}+3 p \beta_{1}\right) \equiv 0
$$

Now, (2.25) and (2.26) lead to

$$
p\left[\alpha_{1}\left(t_{1}+p^{\prime}\right)+\alpha_{2} p\right]-\alpha_{1} p\left(t_{1}+p^{\prime}+3 p \beta_{1}\right) \equiv 0 .
$$

From the definition of $\alpha_{2}$ and (2.27), we deduce

$$
\alpha_{1}^{\prime} \equiv 2 \alpha_{1} \beta_{1}
$$

It follows from (2.28) and the definitions of $t_{1}, \beta_{1}$ that

$$
\alpha_{1}^{3} p^{4} \equiv t_{2}^{2} \mathrm{e}^{2 \alpha}
$$

In the beginning of the proof it was already shown that $t_{2} \not \equiv 0$. Hence, the contradiction here is immediate.

This also completes the proof of Theorem 1.2.

\section{Remarks and a conjecture}

Remark 3.1 Corollary 1.1 or Corollary 1.2 can be strengthened to

$$
N\left(r, \frac{1}{F}\right) \neq S(r, f) .
$$

Remark 3.2 What can be said if ' $p f f^{\prime}-q$ ' is replaced by ' $p f f^{(k)}-q$ ', for any integer $k \geq 2$, in Theorem 1.2?

Remark 3.3 Taking $f(z)=\mathrm{e}^{z}$, we have

$$
N\left(r, \frac{1}{f^{(k)}-a}\right) \sim 2 T(r, f)+S(r, f)
$$

where $k$ is a positive integer, and $a$ is a non-zero constant.

Finally, we present the following more general and quantitative conjecture.

Conjecture 3.1 Let $f$ be a transcendental entire function. Then for any integer $k \geq 1$, and any small function $a(\neq 0)$,

$$
N\left(r, \frac{1}{f^{(k)}-a}\right) \sim 2 T(r, f)+S(r, f) .
$$




\section{Author details}

'Department of Mathematics, China University of Petroleum, Qingdao, 266580, P.R. China. ${ }^{2}$ Department of Mathematics, Nanjing University, Nanjing, 210093, P.R. China.

\section{Acknowledgements}

The authors would like to thank the referee for his/her several important suggestions and valuable comments to our original manuscript which enabled us to improve greatly the quality and readability of the paper.

Received: 22 September 2014 Accepted: 21 April 2015 Published online: 01 May 2015

\section{References}

1. Hayman, WK: Meromorphic Functions. Clarendon, Oxford (1964)

2. Yang, CC, Yi, HX: Uniqueness Theory of Meromorphic Functions. Science Press, Beijing (2003)

3. Hayman, WK: Picard values of meromorphic functions and their derivatives. Ann. Math. (2) 70, 9-42 (1959)

4. Hayman, WK: Research Problems in Function Theory. Athlone Press, London (1967)

5. Mues, E: Über ein Problem von Hayman. Math. Z. 164, 239-259 (1979)

6. Bergweiler, W, Eremenko, A: On the singularities of the inverse to a meromorphic function of finite order. Rev. Mat. Iberoam. 11, 355-373 (1995)

7. Chen, HH, Fang, ML: The value distribution of $f^{n} f^{\prime}$. Sci. China Ser. A 38, 789-798 (1995)

8. Liu, NN, Lü, WR, Shen, TT, Yang, CC: Entire solutions of certain type of difference equations. J. Inequal. Appl. 2014, 63 (2014)

9. Liu, NN, Lü, WR, Yang, CC: On Picard exceptional values of difference polynomials. J. Differ. Equ. Appl. 20, 1437-1443 (2014)

10. Yang $\mathrm{CC}, \mathrm{Hu}, \mathrm{PC} \cdot$ On the value distribution of $\mathrm{ff}^{(\mathrm{k})}$ Kodai Math. J. 19, 157-167 (1996)

11. Yang, CC, Yang, L, Wang, YF: On the zeros of $\left(f^{(k)}\right)^{n} f-a$. Chin. Sci. Bull. 38, 2125-2128 (1993)

12. Bergweiler, W: On the product of a meromorphic function and its derivative. Bull. Hong Kong Math. Soc. 1, 97-101 (1997)

13. Wang, JP: On the zeros of $f^{n}(z) f^{(k)}(z)-c(z)$. Complex Var. Theory Appl. 48, 695-703 (2003)

14. Hennekemper, W: Über die Wertverteilung von $\left(f^{k+1}\right)^{(k)}$. Math. Z. 177, 375-380 (1981)

15. Zhang, DQ: On the value distribution of $\varphi(z) f(z) f^{\prime}(z)$. Acta Math. Sin. 37, 91-98 (1994)

16. Clunie, J: On integral and meromorphic functions. J. Lond. Math. Soc. 37, 17-27 (1962)

17. Bank, S, Laine, I: On the growth of meromorphic solutions of linear and algebraic differential equations. Math. Scand. 40, 116-126 (1977)

18. Laine, I: Nevanlinna Theory and Complex Differential Equations. de Gruyter, Berlin (1993)

19. Mohon'ko, AZ, Mohon'ko, VD: Estimates of the Nevanlinna characteristics of certain classes of meromorphic functions, and their applications to differential equations. Sib. Mat. Zh. 15, 1305-1322 (1974) (in Russian)

20. Hayman, WK, Miles, J: On the growth of a meromorphic function and its derivatives. Complex Var. Theory Appl. 12, 245-260 (1989)

21. He, YZ, Laine, I: The Hayman-Miles theorem and the differential equation $\left(y^{\prime}\right)^{n}=R(z, y)$. Analysis 10, $387-396(1990)$

\section{Submit your manuscript to a SpringerOpen ${ }^{\circ}$ journal and benefit from:}

- Convenient online submission

Rigorous peer review

- Immediate publication on acceptance

- Open access: articles freely available online

- High visibility within the field

- Retaining the copyright to your article 